\title{
Asymptotics of Some Convolutional Recurrences
}

\author{
Edward A. Bender * \\ Department of Mathematics \\ University of California, San Diego \\ La Jolla, CA 92093-0112 \\ ebender@ucsd.edu
}

\author{
Adri B. Olde Daalhuis \\ Maxwell Institute and School of Mathematics \\ The University of Edinburgh \\ Edinburgh, EH9 3JZ, UK
}

A.OldeDaalhuis@ed.ac.uk

\author{
Zhicheng Gao ${ }^{\dagger}$
}

School of Mathematics and Statistics

Carleton University

Ottawa, Ontario K1S5B6

zgao@math. carleton.ca
L. Bruce Richmond* and Nicholas Wormald ${ }^{\ddagger}$
Department of Combinatorics and Optimization
University of Waterloo
Waterloo, Ontario N2L3G1
lbrichmond@uwaterloo.ca, nwormald@uwaterloo.ca

Submitted: Apr 7, 2009; Accepted: Dec 14, 2009; Published: Jan 5, 2010

\begin{abstract}
We study the asymptotic behavior of the terms in sequences satisfying recurrences of the form $a_{n}=a_{n-1}+\sum_{k=d}^{n-d} f(n, k) a_{k} a_{n-k}$ where, very roughly speaking, $f(n, k)$ behaves like a product of reciprocals of binomial coefficients. Some examples of such sequences from map enumerations, Airy constants, and Painlevé I equations are discussed in detail.
\end{abstract}

\section{Main results}

There are many examples in the literature of sequences defined recursively using a convolution. It often seems difficult to determine the asymptotic behavior of such sequences. In this note we study the asymptotics of a general class of such sequences. We prove

\footnotetext{
${ }^{*}$ Research supported by NSERC

${ }^{\dagger}$ Research supported by NSERC

${ }^{\ddagger}$ Research supported by NSERC and Canada Research Chair Program
} 
subexponential growth by using an iterative method that may be useful for other recurrences. By subexponential growth we mean that, for every constant $D>1, a_{n}=o\left(D^{n}\right)$ as $n \rightarrow \infty$. Thus our motivation for this note is both the method and the applications we give.

Let $d>0$ be a fixed integer and let $f(n, k) \geqslant 0$ be a function that behaves like a product of some powers of reciprocals of binomial coefficients, in a general sense to be specified in Theorem 1 . We deal with the sequence $a_{n}$ for $n \geqslant d$ where $a_{d}, a_{d+1}, \cdots, a_{2 d-1} \geqslant$ 0 are arbitrary and, when $n \geqslant 2 d$,

$$
a_{n}=a_{n-1}+\sum_{k=d}^{n-d} f(n, k) a_{k} a_{n-k} .
$$

Without loss of generality,

$$
\text { we assume that } f(n, k)=f(n, n-k)
$$

since we can replace $f(n, k)$ and $f(n, n-k)$ in (1) with $\frac{1}{2}(f(n, k)+f(n, n-k))$.

Theorem 1 proves subexponential growth. Theorem 2 provide more accurate estimates under additional assumptions. In Section 2, we apply the corollary to some examples.

Theorem 1 (Subexponential growth) Let $a_{n}$ be defined by recursion (1) with $a_{d}>0$. Suppose there is a function $R(x)$ defined on $(0,1 / 2]$, an $\alpha>0$ and an $r$ such that

(a) $0<R(x)<r<1$,

(b) $\lim _{x \rightarrow 0+} R(x)=0$,

(c) $0 \leqslant f(n, k)=O\left(n^{-\alpha} R^{k-d}(k / n)\right)$ uniformly for $d \leqslant k \leqslant n / 2$.

Then $a_{n}$ grows subexponentially; in fact,

$$
a_{n}=\left(1+O\left(n^{-\alpha}\right)\right) a_{n-1} .
$$

Proof: We first note that the $a_{n}$ are non-decreasing when $n \geqslant 2 d-1$.

Our proof is in three steps. We first prove that $a_{n}=O\left(C^{n}\right)$ for some constant $C>2$. We then prove that $C$ can be chosen very close to 1 . Finally we deduce (2) and subexponential growth.

First Step: Since the bound in (c) is bounded by some constant times the geometric series $n^{-\alpha} r^{k-d}$ with ratio less than $1, \sum_{k=d}^{n-d} f(n, k)=O\left(n^{-\alpha}\right)$. Hence we can choose $M$ so large that $\sum_{k=d}^{n-d} f(n, k)<1 / 4$ when $n>M$. Next choose $C \geqslant 2$ so large $(C=$ $\max \left\{a_{d}, a_{d+1}, \ldots, a_{2 d-1}, a_{M}, 2\right\}$ will do) that $a_{n}<2 C^{n}$ for $n \leqslant M$. By induction, using the recursion (1), we have for $n>M$

$$
a_{n}<2 C^{n-1}+(1 / 4) 4 C^{n} \leqslant C^{n}+C^{n}=2 C^{n} .
$$


Second Step: By (b) there is a $\lambda$ in $(0,1 / 2)$ such that $R(x)<\frac{1}{2 C}$ for $0<x<\lambda$. Fix any $D \leqslant C$ such that $a_{n}=O\left(D^{n}\right)$, which is true for $D=C$ by the First Step.

Split the sum in (1) into $\lambda n \leqslant k \leqslant(1-\lambda) n$ and the rest, calling the first range of $k$ the "center" and the rest the "tail". Noting $r<1$, the center sum is bounded by

$$
2 \sum_{k=\lambda n+1}^{n / 2} f(n, k) a_{k} a_{n-k}=O\left(D^{n} \sum_{k=\lambda n+1}^{n / 2} r^{k-d}\right)=O\left(\left(r^{\lambda} D\right)^{n}\right) .
$$

Since $a_{j}$ are increasing, the tail sum is bounded by

$$
\begin{aligned}
2 \sum_{k=d}^{\lambda n} f(n, k) a_{k} a_{n-k} & =O\left(n^{-\alpha}\right) a_{n-1} \sum_{k=d}^{\lambda n} R(x)^{k-d} D^{k} \\
& =O\left(n^{-\alpha}\right) a_{n-1} \sum_{k=d}^{\lambda n}(D R(x))^{k-d}=O\left(n^{-\alpha} a_{n-1}\right),
\end{aligned}
$$

where the last equality follows from the fact that $D R(x)<1 / 2$. Combining (3) and (4),

$$
a_{n}=\left(1+O\left(n^{-\alpha}\right)\right) a_{n-1}+O\left(\left(r^{\lambda} D\right)^{n}\right) .
$$

When $r^{\lambda} D>1$, induction on $n$ easily leads to $a_{n}=O\left(\left(r^{\lambda} D^{\prime}\right)^{n}\right)$ for any $D^{\prime}>D$, an exponential growth rate no larger than $r^{\lambda} D^{\prime}$.

Since $r^{\lambda}$ has a fixed value less than one, we can iterate this process, replacing $D$ by $r^{\lambda} D^{\prime}$ at the start of the Second Step. We finally obtain a growth rate $D>1$ with $r^{\lambda} D<1$. This completes the second step.

Third Step: With the value of $D$ just obtained, the last term in (5) is exponentially small and hence is $O\left(n^{-\alpha} a_{n-1}\right)$. Thus we obtain (2) which immediately implies subexponential growth of $a_{n}$, since $1+O\left(n^{-\alpha}\right)<D$ for any $D>1$ and sufficiently large $n$.

To say more than (2), we need additional information about the behavior of the $f(n, k)$. When $f(n, k) / f(n, d)$ is small for each $k$ in the range $d+1 \leqslant k \leqslant n-d-1$, the first and last terms dominate the sum. The following theorem is based on this observation.

Theorem 2 (Asymptotic behavior) Assume (a)-(c) of Theorem 1 hold. Suppose further that there is a $\beta>0$ such that

$$
\frac{f(n, k)}{f(n, d)}=O\left(n^{-\beta} r^{k-d-1}\right) \quad \text { uniformly for } \quad d+1 \leqslant k \leqslant n / 2 .
$$

Then

$$
\log a_{n}=2 a_{d} \sum_{k=2 d+1}^{n} f(k, d)+O\left(\sum_{k=2 d}^{n} f(k, d)\left(k^{-\alpha}+k^{-\beta}\right)\right) .
$$


Proof: We assume $n>2 d$. Remove the $k=d$ and $k=n-d$ terms from the sum in (1). We first deal with the remaining sum. Theorem 1 gives $a_{k}=O\left(D^{k}\right)$ for all $D>1$, so we can assume $D<1 / r$. Using (6)

$$
\begin{aligned}
\sum_{k=d+1}^{n-d-1} f(n, k) a_{k} a_{n-k} & =O\left(f(n, d) n^{-\beta} a_{n-1}\right) \sum_{k=d+1}^{n / 2} r^{k-d-1} D^{k} \\
& =O\left(f(n, d) n^{-\beta} a_{n-1}\right) .
\end{aligned}
$$

Combining this with (1), we obtain

$$
\begin{aligned}
a_{n} & =a_{n-1}+2 a_{d} f(n, d) a_{n-d}+f(n, d) O\left(n^{-\beta}\right) a_{n-1} \\
& =a_{n-1}\left(1+2 a_{d} f(n, d)+\left\{O\left(n^{-\alpha}\right)+O\left(n^{-\beta}\right)\right\} f(n, d)\right),
\end{aligned}
$$

Taking logarithms and noting for expansion purposes that $f(n, d)=O\left(n^{-\alpha}\right)$, we obtain

$$
\log a_{n}-\log a_{n-1}=2 a_{d} f(n, d)+O\left(\left(n^{-\alpha}+n^{-\beta}\right) f(n, d)\right) .
$$

Sum over $n$ starting with $n=2 d+1$. The theorem follows immediately when we note that the constant terms can be incorporated into the $O()$ in $(7)$ since the sum therein is bounded below by a nonzero constant.

Corollary 1 Assume the conditions of Theorem 2 hold and $f(n, d)=\Theta\left(n^{-\alpha}\right)$.

- If $\alpha<1$, then $a_{n}=\exp \left(\Theta\left(n^{1-\alpha}\right)\right)$.

- If $\alpha>1$, then $a_{n}=K+O\left(n^{1-\alpha}\right)$ for some constant $K$.

- If $f(n, d)-A / n$ are the terms of a convergent series, then $a_{n} \sim C n^{2 A a_{d}}$ for some positive constant $C$.

Proof: Since $\alpha>0$ and $\beta>0,(7)$ gives $\log a_{n}=\Theta\left(\sum_{k=2 d+1}^{n} k^{-\alpha}\right)$. The case $\alpha<1$ follows immediately; for $\alpha>1$, we see that $a_{n}$ is bounded and nondecreasing and therefore has a limit $K$. For $m>n$, (2) gives $\log \left(a_{m} / a_{n}\right)=O\left(n^{1-\alpha}\right)$ uniformly in $m$. Letting $m \rightarrow \infty$, we obtain the claim regarding $\alpha>1$.

For $\alpha=1$, the first sum in (7) is $A \log n+B+o(1)$ for some constant $B$, and the last sum in (7) converges.

\section{Examples}

We apply Theorem 2 and Corollary 1 to some recursions which arise from combinatorial applications. In our examples, $f(n, k)$ behaves like a product of the reciprocal of binomial coefficients, which satisfies the conditions of Theorems 1 and 2. A more general case of interest is when $f(n, k)$ takes the form of the product of functions like

$$
g(n, k)=\frac{[a]_{k}[a]_{n-k}}{[a]_{n}}
$$


for some constant $a>0$, where $[x]_{k}=x(x+1) \cdots(x+k-1)=\frac{\Gamma(x+k)}{\Gamma(k)}$, the rising factorial. We note that when $a=1, g(n, k)=\left(\begin{array}{l}n \\ k\end{array}\right)^{-1}$.

We begin with some useful bounds. When $a>0$ and $1 \leqslant k \leqslant n / 2$,

$$
\begin{aligned}
g(n, k) & =\prod_{j=0}^{k-1} \frac{a+j}{a+n-k+j}<\left(\frac{a+k}{a+n}\right)^{k} \\
& \leqslant(k / n)^{k}\left(\frac{1+a / k}{1+a / n}\right)^{k}=O\left((k / n)^{k}\right)=O\left(n^{-1}(3 k / 2 n)^{k-1}\right)
\end{aligned}
$$

since $k(2 / 3)^{k-1}$ is bounded. So $g$ satisfies the condition on $f$ in Theorem $1(\mathrm{c})$, with $\alpha=1$. Similarly, when $a>0$ and $d \leqslant k \leqslant n / 2$,

$$
\frac{g(n, k)}{g(n, d)}=\prod_{j=0}^{k-d-1} \frac{a+d+j}{a+n-k+d+j}=O\left(n^{-1}(3 k / 2 n)^{k-d-1}\right) .
$$

This is in accordance with (6) with $\beta=1$.

Example 1 (Map enumeration constants) There are numbers $t_{n}$ appearing in the asymptotic enumeration of maps in an orientable surface of genus $n$, whose value does not concern us here. Define $u_{n}$ by

$$
t_{n}=8 \frac{[1 / 5]_{n}[4 / 5]_{n-1}}{\Gamma\left(\frac{5 n-1}{2}\right)}\left(\frac{25}{96}\right)^{n} u_{n} .
$$

Then $u_{1}=1 / 10$ and $u_{n}$ satisfies the following recursion [3]

$$
u_{n}=u_{n-1}+\sum_{k=1}^{n-1} f(n, k) u_{k} u_{n-k} \quad \text { for } \quad n \geqslant 2
$$

where

$$
f(n, k)=\frac{[1 / 5]_{k}[1 / 5]_{n-k}}{[1 / 5]_{n}} \frac{[4 / 5]_{k-1}[4 / 5]_{n-k-1}}{[4 / 5]_{n-1}} .
$$

From the observations above, the conditions of Theorem 2 are satisfied with $d=1$, $R(\lambda)=(3 \lambda / 2)^{2}$ and $\alpha=\beta=2$. Hence, $u_{n} \sim K$ for some constant $K$. Unlike the proof in [3], this does not depend on the value of $u_{1}$.

Example 2 (Airy constants) The Airy constants $\Omega_{n}$ are determined by $\Omega_{1}=1 / 2$ and the recurrence [7]

$$
\Omega_{n}=(3 n-4) n \Omega_{n-1}+\sum_{k=1}^{n-1}\left(\begin{array}{l}
n \\
k
\end{array}\right) \Omega_{k} \Omega_{n-k} \text { for } n \geqslant 2 .
$$


Let $\Omega_{n}=n ![2 / 3]_{n-1} 3^{n} a_{n}$. Then $a_{n}$ satisfies (1) with $d=1$ and

$$
f(n, k)=\frac{[2 / 3]_{k-1}[2 / 3]_{n-k-1}}{[2 / 3]_{n-1}} .
$$

Theorem 2 applies with $d=1, R(\lambda)=3 \lambda / 2$ and $\alpha=\beta=1$. Since

$$
f(n, 1)=\frac{1}{n-4 / 3}=\frac{1}{n}+\frac{4 / 3}{n(n-4 / 3)}
$$

and $a_{1}=1 / 6$, we have $a_{n} \sim C n^{1 / 3}$ for some constant $C$.

We note that it is possible to apply the result of Olde Daalhuis [13] to obtain a full asymptotic expansion for $\Omega_{n}$. Let

$$
A_{n}=\frac{\Omega_{n}}{3^{n} n !}
$$

Then the recursion for $\Omega_{n}$ becomes

$$
A_{n}=(n-4 / 3) A_{n-1}+\sum_{k=1}^{n-1} A_{k} A_{n-k}, n \geqslant 2 .
$$

It follows that the formal series

$$
F(z)=\sum_{n \geqslant 1} \frac{A_{n}}{z^{n}}
$$

satisfies the Riccati equation

$$
F^{\prime}(z)+\left(1+\frac{1}{3 z}\right) F(z)-F^{2}(z)-\frac{1}{6 z}=0 .
$$

It then follows from the result of Olde Daalhuis [13] that

$$
A_{n} \sim \frac{1}{2 \pi} \sum_{k=0}^{\infty} b_{k} \Gamma(n-k), \text { as } n \rightarrow \infty,
$$

where $b_{0}=1$ and $b_{k}$ can be computed using the recursion

$$
b_{k}=\frac{-2}{k} \sum_{j=2}^{k+1} b_{k+1-j} A_{j}, \quad k \geqslant 1 .
$$

In particular, we have

$$
\Omega_{n} \sim \frac{1}{2 \pi} \Gamma(n) 3^{n} n !=\frac{1}{2 \pi n}(n !)^{2} 3^{n}, \quad \text { as } n \rightarrow \infty .
$$

It is well known that solutions to the Riccati equation have infinitely many singularities, hence $F(z)$ (via its Borel transform [2]) cannot satisfy a linear ODE with polynomial coefficients. This implies that the sequence $A_{n}$ (and hence $\Omega_{n}$ ) is not holonomic. 
Example 3 The following recursion, with $\ell>0$ and $\ell \neq 1 / 2$, appeared in [6]. The Airy constants are the special case $\ell=1$. The case $\ell=2$ corresponds to the recursion studied in $[9,10]$, which arises in the study of the Wiener index of Catalan trees. We have $C_{1}=\frac{\Gamma(\ell-1 / 2)}{\sqrt{\pi}}$ and, for $n \geqslant 2$,

$$
C_{n}=n \frac{\Gamma(n \ell+(n / 2)-1)}{\Gamma((n-1) \ell+(n / 2)-1)} C_{n-1}+\frac{1}{4} \sum_{k=1}^{n-1}\left(\begin{array}{l}
n \\
k
\end{array}\right) C_{k} C_{n-k} .
$$

Define $a_{n}$ by $C_{n}=n ! g(n) a_{n}$ where $g(1)=1$ and

$$
g(m)=\prod_{k=2}^{m} \frac{\Gamma(k \ell+(k / 2)-1)}{\Gamma((k-1) \ell+(k / 2)-1)} .
$$

Then (11) becomes

$$
a_{n}=a_{n-1}+\sum_{k=1}^{n-1} \frac{g(k) g(n-k)}{4 g(n)} a_{k} a_{n-k},
$$

so $f(n, k)=g(k) g(n-k) / 4 g(n)$.

With $a$ fixed and $x \rightarrow \infty$ and using 6.1.47 on p.257 of [1] (or using Stirling's formula), we have

$$
\begin{aligned}
\frac{\Gamma(x+a)}{\Gamma(x)} & =x^{a}\left(1+\frac{a(a-1)}{2 x}+O\left(1 / x^{2}\right)\right) \\
& =x^{a}\left(1+\frac{a-1}{2 x}\right)^{a}\left(1+O\left(1 / x^{2}\right)\right) \\
& =\left(x+\frac{a-1}{2}\right)^{a}\left(1+O\left(1 / x^{2}\right)\right) .
\end{aligned}
$$

When $m>1$, (13) gives us

$$
\begin{aligned}
g(m) & =\prod_{k=2}^{m}\left(\frac{(2 \ell+1) k-\ell-3}{2}\right)^{\ell}\left(1+O\left(1 / k^{2}\right)\right) \\
& =\Theta(1)\left((\ell+1 / 2)^{m} \prod_{k=2}^{m}\left(k-\frac{\ell+3}{2 \ell+1}\right)\right)^{\ell} \\
& =\Theta(1)\left((\ell+1 / 2)^{m}[a]_{m-1}\right)^{\ell}, \quad \text { where } a=\frac{3 \ell-1}{2 \ell+1} .
\end{aligned}
$$

Hence

$$
f(n, k)=\Theta(1)\left|\frac{[a]_{k-1}[a]_{n-k-1}}{[a]_{n-1}}\right|^{\ell} .
$$

where the absolute values have been introduced to allow for $a<0$. A slight adjustment of the argument leading to (8) and (9) leads to

$$
f(n, k)=O\left(n^{-\ell}(3 k / 2 n)^{\ell(k-1)}\right) \quad \text { and } \quad \frac{f(n, k)}{f(n, 1)}=O\left(n^{-\ell}(3 k / 2 n)^{\ell(k-d-1)}\right)
$$


for $1 \leqslant k \leqslant n / 2$. Hence Theorem 2 applies with $\alpha=\beta=\ell$, and $a_{n}$ converges to a constant when $\ell>1$ by Corollary 1 .

It is interesting to note that there is a simple relation between the sequence $u_{n}$ in Example 1 and the sequence $a_{n}$ in Example 3 with $\ell=2$. It is not difficult to check that the $f(n, k)$ defined in Example 3 is exactly five times the $f(n, k)$ in Example 1: since $a_{1}=5 u_{1}$, we have $a_{n}=5 u_{n}$ for all $n \geqslant 1$. This simple relation suggests a relationship between the number of maps on an orientable surface of genus $g$ and the $g$ th moment of a particular toll function on a certain type of trees. Using a bijective approach, Chapuy [4] recently found an expression for $t_{g}$ as the $g$ th moment of the labels in a random well-labelled tree.

\section{A convolutional recursion arising from Painlevé I}

The following is recursion (44) in [11].

$$
\alpha_{n}=(n-1)^{2} \alpha_{n-1}+\sum_{k=2}^{n-2} \alpha_{k} \alpha_{n-k}, n \geqslant 1, n \geqslant 3 .
$$

It follows from Proposition 14 of [11] that, for $0<\alpha_{1}<1$ and $\alpha_{2}=\alpha_{1}-\alpha_{1}^{2}$,

$$
\alpha_{n}=c\left(\alpha_{1}\right)((n-1) !)^{2}\left(1-\frac{2 \alpha_{2}(n-3)}{3(n-1)^{2}(n-2)^{2}}+\delta_{n}\right),
$$

where $c\left(\alpha_{1}\right)$ depends only on $\alpha_{1}$, and

$$
\delta_{n}=O\left(1 / n^{4}\right)
$$

We note that $\alpha_{n}$ for $n \geqslant 3$ depends only on $\alpha_{2}$. The proof of (15) relies on the fact that $0<\alpha_{2}<1 / 4$ for $0<\alpha_{1}<1$. It is conjectured in [11] that the asymptotic expression (15) actually holds for a wider range of values of $\alpha_{1}$.

For $n \geqslant 1$, let

$$
p_{n}=\frac{\alpha_{n}}{((n-1) !)^{2}} .
$$

Then, as shown in [11], $p_{n}$ satisfies recursion (1) with $d=2$ and

$$
f(n, k)=\left(\frac{(n-k-1) !(k-1) !}{(n-1) !}\right)^{2}
$$

We note here $f(n, 2)=O\left(n^{-4}\right)$. It follows from Theorem 2 that

$$
p_{n}=p\left(1+\epsilon_{n}\right) \text { for any } \alpha_{2}>0,
$$

where $p=p\left(\alpha_{2}\right)$ is a positive constant and $\epsilon_{n}=O\left(1 / n^{3}\right)$. 
It is also interesting to note that, with $\alpha_{1}=1 / 50, \alpha_{2}=49 / 2500$, the sequence $\alpha_{n}$ is related to the sequence $u_{n}$ in Example 1 by

$$
\alpha_{n}=[1 / 5]_{n}[4 / 5]_{n-1} u_{n}
$$

As mentioned in [11], the formal series $v(t)=\sum_{n \geqslant 1} \alpha_{n} t^{-n}$ satisfies

$$
t^{2} v^{\prime \prime}+t v^{\prime}-\left(t+2 \alpha_{1}\right) v+t v^{2}+\alpha_{1}=0
$$

and hence, with

$$
t=\frac{8 \sqrt{6}}{25} x^{5 / 2}
$$

$y(x)=(x / 6)^{1 / 2}(1-2 v(t))$ satisfies the following Painlevé I:

$$
y^{\prime \prime}=6 y^{2}-x
$$

This connection with Painlevé I is used in [8] to show that the sequence $\alpha_{n}$ is not holonomic (It follows that $u_{n}$ and $t_{n}$ in Example 1 are also not holonomic). The proof uses the fact that solutions to Painlevé I have infinitely many singularities and hence cannot satisfy a linear ODE with polynomial coefficients.

In the following we apply the techniques of [14] to prove that (15) holds for any complex constant $\alpha_{1}$. It is convenient to introduce the formal series

$$
u_{0}(z)=v\left(z^{2}\right)=\sum_{n=2}^{\infty} b_{n} z^{-n}=\sum_{n=1}^{\infty} \alpha_{n} z^{-2 n}
$$

It follows from (16) that $u=u_{0}(z)$ is a formal solution to the differential equation

$$
\frac{1}{4} u^{\prime \prime}+\frac{1}{4 z} u^{\prime}-\left(1+\frac{2 \alpha_{1}}{z^{2}}\right) u+u^{2}+\frac{\alpha_{1}}{z^{2}}=0 .
$$

The Stokes lines for this differential equation are the positive and the negative real axes. When the negative real axis is crossed the Stokes phenomenon switches on a divergent series

$$
u_{1}(z)=K e^{2 z} z^{-1 / 2} \sum_{n=0}^{\infty} c_{n} z^{-n}
$$

in which the Stokes multiplier $K$ is a constant (depending on the constant $\alpha_{1}$ ). To determine the coefficients $c_{n}$ we observe that $u_{1}$ is a solution of the linear differential equation

$$
\frac{1}{4} u_{1}^{\prime \prime}+\frac{1}{4 z} u_{1}^{\prime}-\left(1+\frac{2 \alpha_{1}}{z^{2}}-2 u_{0}\right) u_{1}=0 .
$$

Hence, for the coefficients $c_{n}$ we can take $c_{0}=1$ and for the others we have

$$
n c_{n}=\frac{1}{4}\left(n-\frac{1}{2}\right)^{2} c_{n-1}+2 \sum_{k=4}^{n+1} b_{k} c_{n+1-k}, \quad n \geqslant 1 .
$$


The first five coefficients are

$$
c_{0}=1, \quad c_{1}=\frac{1}{16}, \quad c_{2}=\frac{9}{512}, \quad c_{3}=\frac{75}{8192}+\frac{2}{3} \alpha_{2}, \quad c_{4}=\frac{3675}{524288}+\frac{13}{24} \alpha_{2} .
$$

In a similar manner it can be shown that when the positive real axis is crossed the Stokes phenomenon switches on a divergent series

$$
u_{2}(z)=i K e^{-2 z} z^{-1 / 2} \sum_{n=0}^{\infty}(-1)^{n} c_{n} z^{-n} .
$$

This is all the information that is needed to conclude that

$$
\alpha_{n}=b_{2 n} \sim \frac{K}{\pi} \sum_{k=0}^{\infty}(-1)^{k} c_{k} \frac{\Gamma\left(2 n-k-\frac{1}{2}\right)}{2^{2 n-k-(1 / 2)}}, \quad \text { as } n \rightarrow \infty .
$$

By taking the first 4 terms in this expansion we can verify that (15) holds for any complex constant $\alpha_{1}$.

For more details see [12], [13] and [14]. (It's best to get the version of the first reference on the website http://www.maths.ed.ac.uk/ adri/public.htm.)

Acknowledgement We would like to thank Philippe Flajolet for bringing our attention to references [5] and [7]

\section{References}

[1] M. Abramowitz and I.A. Stegun, Handbook of Mathematical Functions With Formulas, Graphs and Mathematical Tables, National Bureau of Standards, Applied Mathematics Series - 55 (1964). Available online at http://www.math.sfu.ca/ cbm/aands/ and other sites.

[2] W. Balser, From divergent series to analytic functions, Springer-Verlag Lecture Notes, No 1582 (1994)

[3] E.A. Bender, Z.C. Gao and L.B. Richmond, The map asymptotics constant $t_{g}$, Electron. J. Combin. 15 (2008), R51.

[4] G. Chapuy, The structure of unicellular maps, and a connection between maps of positive genus and planar labelled trees, preprint, 2009.

[5] J. A. Fill , P. Flajolet, and N. Kapur, Singularity analysis, Hadamard products, and tree recurrences, J. Comput. Appl. Math. 174 (2005), 271-313.

[6] J. A. Fill and N. Kapur, Limiting distributions for additive functionals, Theoret. Comput. Sci. 326 (2004), 69-102.

[7] P. Flajolet and G. Louchard, Analytic variations on the Airy distribution, Algorithmica 31 (2001), 361-377.

[8] S. Garoufalidis, T. T. Lê, and Marcos Mariño , Analyicity of the free energy of a closed 3-manifold, preprint, 2008. 
[9] S. Janson, The Wiener index of simply generated random trees, Random Struct. Alg. 22 (2003), 337-358.

[10] S. Janson and P. Chassaing, The center of mass of the ISE and the Wiener index of trees, Elect. Comm. in Probab. 9 (2004), 178-187.

[11] N. Joshi and A.V. Kitaev, On Boutroux's Tritronquée Solutions of the First Painleveé Equation, Studies in Applied Math 107 (2001), 253-291.

[12] Olde Daalhuis, A. B., Hyperasymptotic solutions of higher order linear differential equations with a singularity of rank one. Proc. R. Royal Soc. Lond. A 454, 1-29, (1998).

[13] Olde Daalhuis, A. B., Hyperasymptotics for nonlinear ODEs I: A Ricatti equation, Proc. Royal Soc. Lond. A. 461, 2503-2520, (2005).

[14] Olde Daalhuis, A. B., Hyperasymptotics for nonlinear ODEs II: The first Painlevé equation and a second-order Riccati equation, Proc. Royal Soc. Lond. A. 461, 30053021, (2005). 